\title{
Acercamiento al manejo del zorro andino (Lycalopex culpaeus) en el altiplano Norte de Bolivia. Análisis arqueozoológicos en los períodos Formativo-Wancarani y Horizonte Medio-Tiwanaku
}

\section{Management of the andean fox (Lycalopex culpaeus) on the northern Bolivian highlands. An archaeozoological approach from the Formativo-Wancarani and Horizonte Medio-Tiwanaku periods}

\author{
VELIA V. MENDOZA ESPAÑA \\ Universidad Mayor de San Andrés, Laboratorio de Zooarqueología. \\ email: velia_arqueologia@yahoo.com
}

(Received 15 May 2018; Revised 10 June 2018; Accepted 6 August 2018)

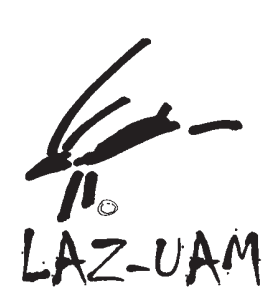

RESUMEN: El presente artículo muestra los resultados de la identificación del manejo del zorro andino en el Altiplano norte de Bolivia para los períodos Formativo-Wancarani (2000 a.C.-400 d.C.) y Horizonte medio-Tiwanaku (400-1.100 d.C.), para lo cual se realizó el análisis de restos arqueofaunísticos y representaciones iconográficas, apoyado con algunos datos históricos. El zorro andino, tanto en la sociedad Wancarani como en el Estado Tiwanaku, tuvo un manejo ritual, su presencia en contextos domésticos (ofrenda de construcción), funerarios y ceremoniales nos habla de un uso simbólico complejo relacionándolo, en el caso de Tiwanaku, con la presencia de un personaje de élite que podría ser un sacerdote. Esto nos llevaría a afirmar que en época prehispánica el zorro andino era considerado un animal sagrado. Este rol cambió en la colonia, ya que la intromisión de fauna alóctona (ovino, porcino y gallinas, entre otros), accesible a este cánido, lo convirtió en un depredador incontrolable perseguido y odiado por las comunidades indígenas.

PALABRAS CLAVE: ALTIPLANO NORTE DE BOLIVIA, ZORRO ANDINO, TIWANAKU, FORMATIVO, MANEJO RITUAL

\begin{abstract}
This article shows the results of the identification of the management of the Andean fox (Lycalopex culpaeus) in the northern Altiplano of Bolivia for the periods Formative-Wancarani (2000 BC-400 AD) and Middle Horizon-Tiwanaku (400-1100 AD). For which the analysis of archaeofauna remains and iconographic representation was carried out, supported with some historical data. The Andean fox had a ritual handling in the Wancarani society as in the Tiwanaku State showed thought domestic, funerary and ceremonial contexts that tells us the complex symbolic use relating it. In case of Tiwanaku, it's the presence of a character of elite, who could be a priest. All of this would lead us to affirm that in prehispanic times the fox was considered a sacred animal. This role changed in colony, since the intromission of allochthonous fauna, accessible to this canid (sheep, swine and chickens, among others), turned it into a predator persecuted and hated by the indigenous communities.
\end{abstract}




\section{INTRODUCCIÓN}

Estudios del zorro andino en contextos arqueológicos son muy escasos, lo que se debe tal vez a la difícil identificación de sus restos óseos con respecto al perro, más aún cuando no se cuenta con elementos óseos diagnósticos (cráneo, mandíbulas y piezas dentales). Existen muy pocos reportes de la presencia de zorro andino en diferentes contextos arqueológicos de Bolivia (Paz et al., 2008; Albarracín-Jordán et al., 2014; Capriles, 2017) mientras que, en Sudamérica el trabajo de Wing (1989) demuestra la presencia del zorro andino asociado a entierros humanos en el Precerámico de la costa de Ecuador y Perú.

El zorro andino (Lycalopex culpaeus) es un cánido considerado el más grande dentro de las especies de zorros sudamericanos y se caracteriza por tener el rostro más angosto, la cabeza, cuello, orejas y patas de color rojizo. Presenta una cola peluda de gran longitud y puede llegar a medir un metro de largo y pesar $9 \mathrm{~kg}$. Se alimentan de frutos, pequeños animales vertebrados e invertebrados, carroña, huevos y plantas. Las hembras pueden reproducirse cuando llegan al año de edad, la gestación dura de 52 a 67 días, y llegan a tener hasta 8 crías (Mercado \& Millares, 1999; Deustua, 2008).

En Bolivia existen seis especies silvestres de cánidos: Lycalopex gymnocercus, Lycalopex culpaeus, Speothos venaticus, Chrysocyon brachyurus, Atelocynus microtis, Cerdocyon thous (Wallace et al., 2010) y una doméstica, Canis lupus familiaris.

El problema de esta investigación parte de la interrogante: ¿Cómo fue el manejo del zorro andino en los períodos Formativo-Wancarani y Horizonte medio-Tiwanaku en el Altiplano norte de Bolivia?, se intentó contestar a esta interrogante con los únicos restos óseos diagnósticos que corresponden a tres individuos de zorro andino. Los mismos provienen del montículo de Chuquiña (Departamento de Oruro), templo de Putuni, en el sitio de Tiwanaku, y Miraflores, en la ciudad de La Paz, (Departamento de La Paz); y tres artefactos con representaciones iconográficas de este animal: una tableta de rapé del sitio de Niño Korin; una estatuilla encontrada en la pirámide de Akapana en el sitio de Tiwanaku, ambas ubicadas dentro del Departamento de La Paz, y una estatuilla sin proveniencia.

De esta manera el objetivo de la investigación fue identificar las características de manejo del zorro andino, para lo cual se realizó el análisis ar- queozoológico e iconológico de restos arqueofaunísticos y representaciones iconográficas, apoyado con algunos datos históricos.

\section{CONTEXTO ARQUEOLÓGICO}

Chuquiña se encuentra en la Provincia Cercado del Departamento de Oruro, la cual abarca la región ecológica altiplano semihúmedo. El sitio arqueológico corresponde a un montículo ubicado dentro de los límites de la Empresa Minera Inti Raymi, a dos kilómetros al este del río Desaguadero. El montículo tiene filiación Wankarani (2000 a.C. a 400 d.C.) que corresponde al periodo Formativo. Dentro de este montículo, el año 2003 en el marco del Proyecto Orureño Formativo, se encontró el cráneo de zorro en un área que corresponde a una superficie de ocupación y a una, profundidad de $445 \mathrm{~cm}$.

Por su parte, Putuni es un templo que se encuentra ubicado al noroeste del sitio arqueológico, Centro Cívico Ceremonial y Residencial de Tiwanaku el cual pertenece a la Provincia Ingavi, Departamento de La Paz. La estructura es de base rectangular compuesta por sillares de andesita gris y arenisca y está conformada por una plataforma de $6 \mathrm{~m}$ que rodea el área templaria, permitiendo en su interior una plaza semi hundida donde están localizados restos de cámaras que conforman los contornos. Su construcción se dio entre el 500 y 1000 d.C., perteneciendo a las denominadas élites Tiwanaku (400 al 1100 d.C.).

Los restos arqueofaunísticos de zorro fueron excavados dentro del Proyecto Arqueológico Putuni 2009, hallados en la cuarta cámara y en asociación con tres individuos humanos (adulto, joven e infante), restos de madera carbonizada probablemente queñua y huesos de camélido que presentaban marcas de corte y alteraciones térmicas (Viviani \& Callisaya, 2009).

En cuanto al sitio arqueológico de Miraflores (Av. Bush), éste se encuentra en la ciudad de La Paz, su cronología corresponde a Tiwanaku (4001100 d.C.). Los restos arqueofaunísticos de zorro andino fueron identificados al realizar trabajos de monitoreo y rescate para la actual construcción de la Línea Blanca del Teleférico. Estos restos se encontraron en las capas inferiores de un «bolsón votivo» y no presentó otro material asociado (Lemuz, 2016). 
Los sitios arqueológicos de donde provienen las representaciones iconográficas de zorros andinos (estatuillas y tableta de rapé) corresponden cronológicamente al período Tiwanaku (400-1100 d.C.) y provienen de los sitios: Tiwanaku (pirámide de Akapana) y Niño Korin (Charazani) (Figura 1). La otra estatuilla de metal no tiene procedencia específica y actualmente se encuentra en los depósitos del Museo Nacional de Arqueología de la ciudad de La Paz. La misma se consideró en el análisis debido a que son muy escasas las representaciones de zorro y además ésta es muy similar en materia prima, tamaño y morfología a la estatuilla de Akapana.

Akapana es una pirámide ubicada en el Centro Cívico y Ceremonial de Tiwanaku. Se considera la estructura más imponente, tiene una altura de 16,50 m, su longitud máxima es de $203 \mathrm{~m}$ y el ancho máximo (E a O) es de $192 \mathrm{~m}$. La planta tiene forma de la mitad de una cruz andina, los muros tienen revestimiento de arenisca, tanto pilares como silla- res (Manzanilla, 1992). Posee siete plataformas y pequeñas estructuras y diferentes tipos constructivos asociados.

La estatuilla de metal con representación de zorro se encontró en un cuarto en la cima de la pirámide. La misma fue parte de una ofrenda, posterior al desmantelamiento de la estructura, que constaba de huesos de camélidos, cerámica doméstica y ritual, madera, artefactos de hueso, artefactos de plata y cobre, restos de frutos y plantas tropicales. Además se registró la existencia de fuego en la parte central del apisonado donde se dispuso la ofrenda (Manzanilla, 1992).

El sitio conocido como Niño Korin, se encuentra en la región de Charazani que es un valle mesotermo del altiplano norte del Departamento de La Paz (Figura 1). En la comunidad de Callijchu se reportó el hallazgo de un paquete ritual que fue estudiado anteriormente (Cordero, 1967; Oblitas, 1978; Mendoza, 2004), siendo parte de este la tableta de rapé con representación de zorro, asociada

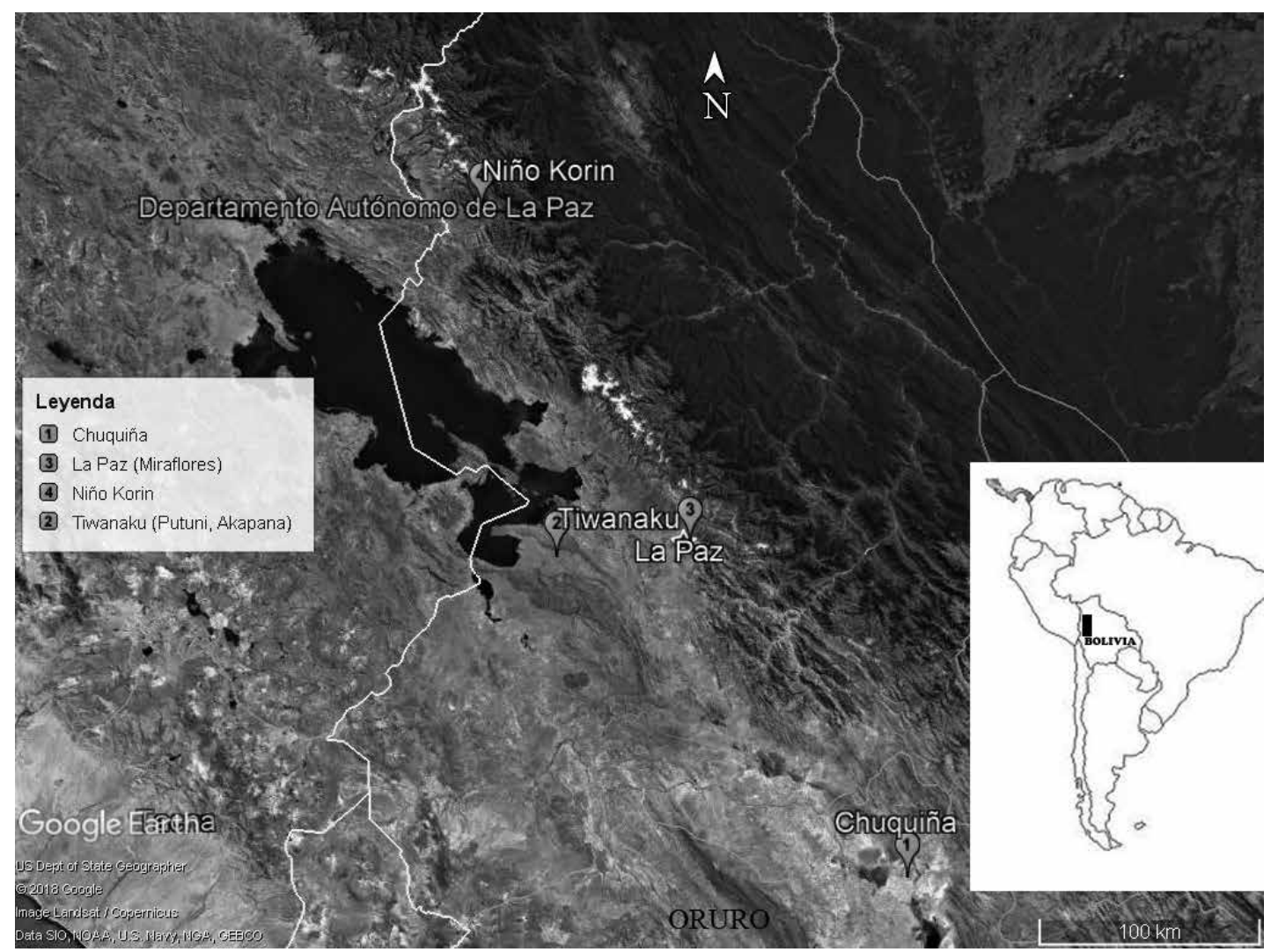

FIGURA 1

Ubicación de los restos arqueofaunísticos y representaciones iconográficas de zorro andino (Modificado de Google Earth, 07/2018). 


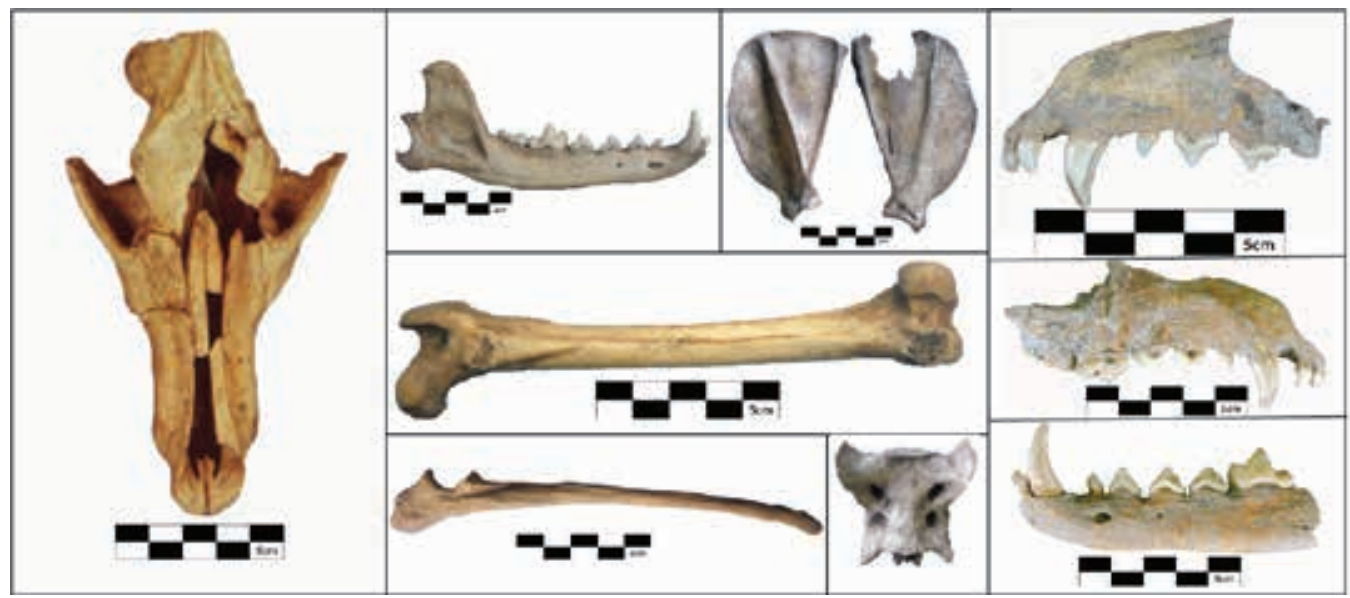

FIGURA 2

A) Cráneo de zorro de Chuquiña. B) Mandíbula y parte del esqueleto postcraneal de zorro de Putuni. C) Cráneo y mandíbula de zorro de Miraflores.

a otra tableta, una cucharita, un poro, un mortero de madera, cuatro manos de mortero hechos también de madera y fragmentos de caña hueca.

\section{MATERIAL Y MÉTODOS}

El análisis arqueofaunístico se llevó a cabo en el Laboratorio de Zooarqueología de la carrera de Antropología-Arqueología de la Universidad Mayor de San Andrés. Este análisis consistió en el ensamblaje de los restos, identificación anatómica, taxonómica, determinación de edad y sexo, osteometría y análisis tafonómico.

1. Zorro de Chuquiña (Figura 2A). El cráneo carece, en parte, del neurocráneo, los arcos cigomáticos se encuentran rotos, la parte ventral también está incompleta, el hueso nasal está fragmentado, el maxilar derecho e izquierdo no cuentan con dos incisivos y tres premolares.

2. Zorro del templo de Putuni (Figura 2B). Carece de cráneo, las mandíbulas y el resto del esqueleto se encuentran en buen estado de conservación. El esqueleto postcraneal comprende escápulas, cúbitos, radios, metacarpos, vértebras cervicales, sacro, fémur, metatarsos, tarsos y falanges.

3. Zorro de Miraflores (Figura 2C). Comprende los premaxilares, maxilares y mandíbulas fragmentadas derechas e izquierdas. Las pie- zas dentales de las mandíbulas se encuentran incompletas.

4. Estatuilla de metal de la pirámide de Akapana (Figura 4). La estatuilla de cobre con la representación naturalista de un zorro sentado está fabricada con la técnica de molde, mide $4,9 \mathrm{~cm}$ de largo, $2,78 \mathrm{~cm}$ de alto y su grosor máximo es de $1,7 \mathrm{~cm}$.

5. Tableta de rapé del sitio Niño Korin (Figura 5). La tableta de rapé con representación de un zorro andino es de madera y presenta forma hiperbólica según los grupos morfológicos que sugiere Llagostera et al. (1988). Sus dimensiones son: largo $18 \mathrm{~cm}$, ancho máximo $7 \mathrm{~cm}$, ancho mínimo $5,4 \mathrm{~cm}$, grosor máximo $1 \mathrm{~cm}$ y grosor mínimo $0,5 \mathrm{~cm}$ (Mendoza, 2004).

6. Estatuilla de zorro descontextualizada (Figura 3). Es idéntica en materia prima y dimensiones a la estatuilla de zorro de la pirámide de Akapana pero posee más pátina.

La identificación taxonómica de los restos arqueofaunísticos se llevó a cabo mediante la comparación con las colecciones de referencia de la Colección Boliviana de Fauna (CBF) y Laboratorio de Zooarqueología de la UMSA. La estimación de edad se determinó a partir de los parámetros establecidos para perros prehispánicos (Valadez, 1996 a, b; Blanco et al., 2009) tomando en cuenta las variables del desgaste dental, fusión de las suturas craneales y epifización del esqueleto postcraneal. La determinación de sexo estuvo sujeta tam- 


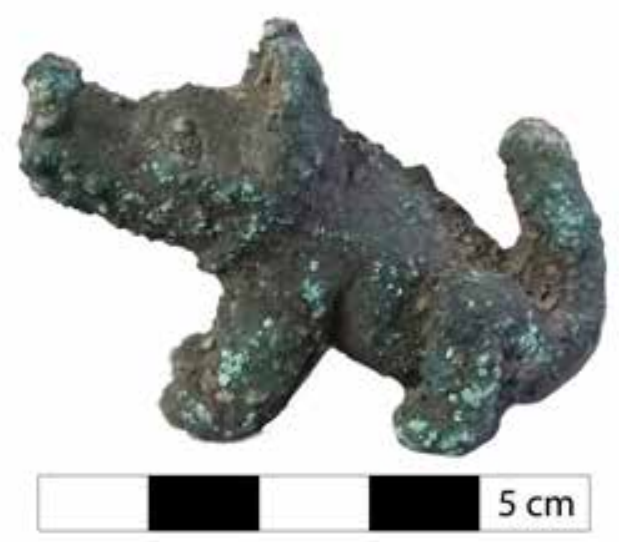

FIGURA 3

Estatuilla de zorro andino sin contexto.

bién a los parámetros que se tienen para perros, determinados por Blanco et al. (2009). También se realizó la osteometría (Driesch, 1976; Meadow, 2000; Blanco et al., 2009) para determinar la talla y diferenciar zorros andinos de otras especies que son más pequeñas como Speothos venaticus y Lycalopex gymnocercus (De Mello \& Zuercher, 2005; Lucherini \& Luengos, 2008; Guzmán et al., 2009; Wallace et al., 2010).

El análisis tafonómico se realizó con una lupa de diferentes aumentos y los resultados de las marcas de cortes del cráneo de Chuquiña se compararon con el trabajo de Pérez Ripoll \& Morales Pérez (2008).

En el caso de la iconografía, se amplió el análisis iconológico de zorro andino llevado a cabo por Mendoza (2004). El mismo adoptó la metodología planteada por Hocquenghem (1987) basada en el método Panofsky.

\section{RESULTADOS Y DISCUSIÓN}

Los tres zorros andinos fueron identificados a partir de la comparación con colecciones de referencia actual de esta especie y otros cánidos que se encuentran disponibles en la Colección Boliviana de Fauna y el Laboratorio de Zooarqueología, to-

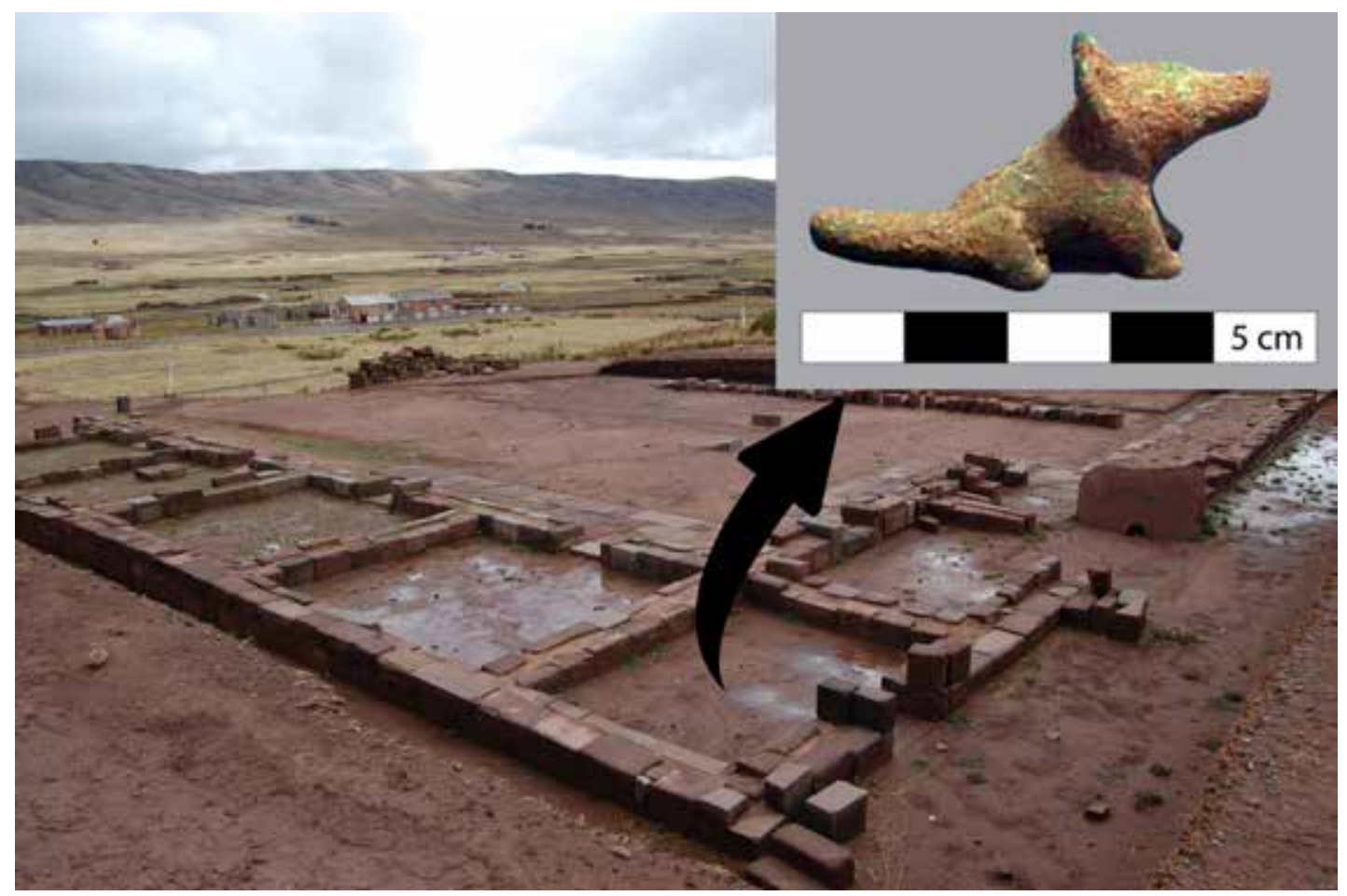

FIGURA 4

Habitación en la cima de la pirámide de Akapana donde se excavó la ofrenda con la estatuilla de zorro andino (Fotografía original de Akapana de Irene Delaveris, 2009). 


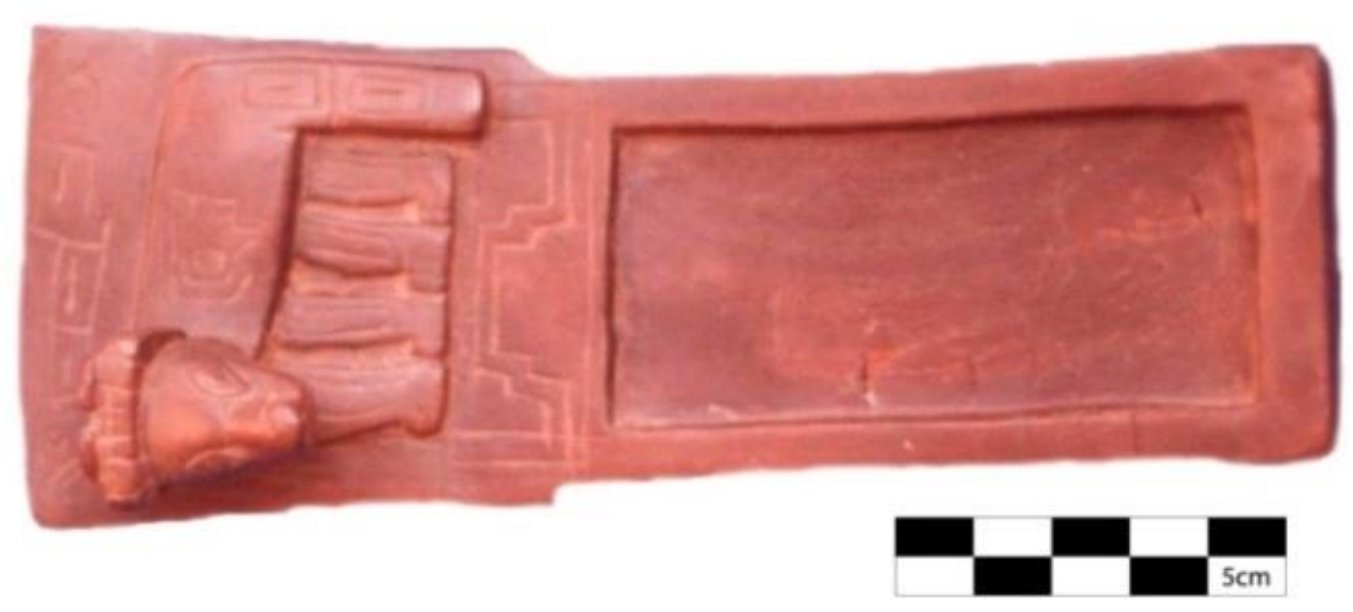

FIGURA 5

Tableta de rapé con representación de un zorro andino.

mando como criterios la morfología y osteometría (mediciones disponibles en la literatura especializada).

El zorro de Chuquiña corresponde a un macho adulto (aprox. 6 años) (Tablas 1 y 2). El cráneo presenta marcas de raspado, pequeños cortes y una profunda incisión, evidenciando que fue despellejado y descarnado para extraer la piel. Posteriormente fue ofrendado y enterrado en el subsuelo de una vivienda como una ofrenda de construcción (cadáver de un animal que se ofrece a la Pachamama o madre tierra al iniciar el cimiento de una construcción para que esta llegue a buen término). Lo que denota un manejo ritual-simbólico, como sucede con cráneos de perros ofrendados en los cimientos de estructuras formativas (Plaza, 1998; Goepfert, 2004; Mauricio Uribe, Comunicación personal).

El zorro de Putuni corresponde también a un macho adulto (aprox. 7 años) (Tablas 3, 4 y 5) que fue colocado como acompañante de personajes humanos de élite que fueron enterrados en un sarcófago del templo. Pudo estar como parte del ajuar o como ofrenda a estos. Este esqueleto corresponde a

\begin{tabular}{|l|c|c|c|c|c|c|}
\cline { 2 - 7 } \multicolumn{1}{c|}{} & Longitud palatal & Ancho máximo palatal & Ancho interorbital & Ancho frontal & Longitud facial & Ancho mínimo frontal \\
\hline Zorro de Chuquiña & 83 & 45,5 & 27,8 & 35 & 82,3 & 23,5 \\
\hline
\end{tabular}

TABLA 1

Medidas craneales en milímetros del zorro de Chuquiña.

\begin{tabular}{|l|c|c|c|c|c|c|c|c|c|c|}
\cline { 2 - 12 } \multicolumn{1}{c|}{} & \multicolumn{4}{c}{ Derecho } & \multicolumn{4}{c|}{ Izquierdo } \\
\cline { 2 - 13 } \multicolumn{1}{c|}{} & I3 & C & PM4 & M1 & M2 & I3 & C & PM3 & M1 & M2 \\
\hline Altura & 5 & 14 & 8,3 & 5 & 3,2 & Raíz & Raíz & 6,8 & 5 & 3,5 \\
\hline Ancho & & 4,8 & & 14 & 10,5 & “ & “ & 3,8 & & 10,5 \\
\hline Longitud & 3,8 & 7 & 16 & 10 & 6 & “ & “ & & 9,1 \\
\hline
\end{tabular}

TABLA 2

Medidas dentales en milímetros del zorro de Chuquiña.

\begin{tabular}{|c|c|c|c|c|c|}
\cline { 2 - 5 } \multicolumn{1}{c|}{} & $\begin{array}{l}\text { Longitud dentario } \\
\text { Id-proceso angular }\end{array}$ & Altura mandíbula & Altura rama dentario & Altura a nivel M1 & $\begin{array}{l}\text { Longitud mandíbula } \\
\text { Id-cóndilo }\end{array}$ \\
\hline Dentario izquierdo & 123,6 & 16,8 & 46,9 & 19,4 & 122,8 \\
\hline
\end{tabular}

TABLA 3

Medidas en milímetros del dentario del zorro de Putuni. 


\begin{tabular}{|c|c|c|c|c|c|c|c|c|c|c|c|c|c|c|c|c|c|c|c|c|}
\hline & \multicolumn{11}{|c|}{ Derecho } & \multicolumn{9}{|c|}{ Izquierdo } \\
\hline & $\mathbf{I}_{1}$ & $I_{2}$ & $\mathbf{I}_{3}$ & C & $\mathrm{Pm}_{1}$ & $\mathrm{Pm}_{2}$ & $\mathrm{Pm}_{3}$ & $\mathbf{P M}_{4}$ & $\mathbf{M}_{1}$ & $\mathbf{M}_{2}$ & $\mathbf{M}_{3}$ & $\mathbf{I}_{3}$ & $\mathbf{C}$ & $\mathbf{P m}_{1}$ & $\mathrm{Pm}_{2}$ & $\mathrm{Pm}_{3}$ & $\mathbf{P M}_{4}$ & $\mathbf{M}_{1}$ & $\mathbf{M}_{2}$ & $\mathbf{M}_{3}$ \\
\hline Altura & 2,2 & & 3,8 & 17,1 & 3,5 & 5,4 & 5,9 & 6,0 & 11 & 4 & 3,2 & 4,2 & 18 & 3,0 & 6,2 & 6,8 & 6,1 & 10,4 & 4,3 & \\
\hline Ancho & 2,1 & & 3,6 & 5,3 & 2,5 & 3,2 & 3,5 & 4,5 & 7,2 & 5,8 & 4,1 & 3,9 & 5,4 & 2,6 & 3 & 3,6 & 4,4 & 6,7 & 6,1 & \\
\hline Longitud & 2,5 & $(2,5)$ & 3,4 & 9,1 & 4,2 & 8 & 9,4 & 9,5 & 15,9 & 8 & 4 & 2,6 & 8,7 & 3,6 & 7,8 & 9,5 & 9,6 & 15,7 & 8,5 & $(3,6)$ \\
\hline
\end{tabular}

TABLA 4

Medidas en milímetros de piezas dentales del zorro de Putuni.

\begin{tabular}{|c|c|c|c|c|c|c|c|c|c|c|c|c|c|c|c|}
\hline & HS & SLC & GLP & GL & BPC & Bp & Bd & SD & GLC & DC & GB & BPacr & GLPa & BPacd & PL \\
\hline Escápula I & 96,7 & 18,7 & 21,4 & & & & & & & & & & & & \\
\hline Cúbito & & & & 146,2 & 130 & & & & & & & & & & \\
\hline Radio & & & & 124,2 & & 14,1 & 18,1 & 9,5 & & & & & & & \\
\hline Metacarpo $1 \mathrm{D} / \mathrm{I}$ & & & & $43 / 44,1$ & & & $8 / 7,4$ & & & & & & & & \\
\hline Metacarpo II D/I & & & & $51 / 51,1$ & & & $64 / 7$ & & & & & & & & \\
\hline Metacarpo III D/I & & & & $51,7 / 50,7$ & & & $6,8 / 6,4$ & & & & & & & & \\
\hline Metacarpo IV D/I & & & & 43,3 & & & 7,8 & & & & & & & & \\
\hline Fémur D & & & & 138,8 & & 29,6 & 26,2 & 10,7 & 140,4 & 14,1 & & & & & \\
\hline Metatarso 1 & & & & 57,9 & & & 6,8 & & & & & & & & \\
\hline Metatarso II & & & & 63,1 & & & 6,7 & & & & & & & & \\
\hline Metatarso III & & & & 61,5 & & & 6,9 & & & & & & & & \\
\hline Metatarso IV & & & & 54,4 & & & 8,2 & & & & & & & & \\
\hline Calcáneo D/I & & & & $35,5 / 35,5$ & & & & & & & $15,6 / 15,7$ & & & & \\
\hline Astrágalo D/I & & & & $21,6 / 21,7$ & & & & & & & & & & & \\
\hline Falanges 1ras & & & & $\begin{array}{c}22,7 ; 22,7 ; \\
21,3 ; 22,4 ; \\
20,0 ; 19,8 ; \\
17,8\end{array}$ & & $\begin{array}{c}6.5 ; 7,0 \\
6,5 ; 6,7 \\
6,5 ; 6,6 ; \\
7,1\end{array}$ & $\begin{array}{c}5,4 ; 5,4 ; \\
5,6 ; 5,5 ; \\
5,7 ; 5,7 \\
; 5,8\end{array}$ & $\begin{array}{c}3,4 ; 3,5 ; \\
3,6 ; 3,7 ; \\
3,6 ; 3,5 ; \\
4,0\end{array}$ & & & & & & & \\
\hline Falanges 2das & & & & $\begin{array}{c}16,4 ; 16,1 ; \\
16,5\end{array}$ & & $\begin{array}{c}5,7 ; 6,0 \\
5,6\end{array}$ & $\begin{array}{c}4,9 ; 5,2 ; \\
5,0\end{array}$ & $\begin{array}{c}3,4 ; 3,9 ; \\
3,2\end{array}$ & & & & & & & \\
\hline Cervical 3 & & & & & & & & & & & & 21,7 & 30,3 & 24,6 & \\
\hline Cervical 4 & & & & & & & & & & & & 23,5 & 22,4 & 20,4 & \\
\hline Cervical 5 & & & & & & & & & & & & 23,9 & 30,3 & 23,1 & \\
\hline Sacro & & & & 29,7 & & & & & & & 32,6 & & & & 27 \\
\hline
\end{tabular}

TABLA 5

Medidas en milímetros del esqueleto post craneal del zorro de Putuni.

\begin{tabular}{|c|c|c|c|c|c|c|c|c|c|c|c|c|c|}
\hline & \multicolumn{3}{|c|}{ Derecho } & \multicolumn{3}{|c|}{ Izquierdo } & & \multicolumn{3}{|c|}{ Derecho } & \multicolumn{3}{|c|}{ Izquierdo } \\
\hline & Altura & Ancho & Long. & Altura & Alto & Long. & & Altura & Ancho & Long. & Altura & Alto & Long. \\
\hline $\mathbf{I}^{1}$ & 3,8 & 2,9 & 2,6 & 3,4 & 3,1 & 2,9 & $I_{1}$ & - & - & - & - & - & - \\
\hline $\mathbf{I}^{2}$ & 4,9 & 3,7 & 2,9 & 4,7 & 3,7 & 2,9 & $I_{2}$ & - & - & - & - & - & - \\
\hline $\mathbf{I}^{3}$ & 7,4 & 4,6 & 3,4 & 7,2 & 4,8 & 3,4 & $I_{3}$ & - & - & - & 5,7 & 3,4 & 3,3 \\
\hline $\mathrm{C}$ & $(14,5)$ & 5 & 7,8 & $(14,3)$ & 5,1 & 8 & $\mathrm{C}$ & 18,9 & 5,5 & 9,4 & 18,5 & 5,5 & 8,7 \\
\hline $\mathbf{P m}^{1}$ & 4,2 & 2,6 & 3,9 & 4,4 & 2,8 & 4 & $\mathbf{P m}_{1}$ & - & - & - & 3,9 & 2,2 & 3,9 \\
\hline $\mathbf{P m}^{2}$ & 6,3 & 3,1 & 8,8 & 6,3 & 3,2 & 8,6 & $\mathbf{P m}_{2}$ & - & - & - & 7 & 3,1 & 8 \\
\hline $\mathbf{P m}^{3}$ & 6,6 & 3,5 & 10,2 & 6 & 3,5 & 10,4 & $\mathbf{P m}_{3}$ & 7,4 & 3,3 & 9,1 & 6,7 & 3,5 & 8,7 \\
\hline $\mathbf{P m}^{4}$ & 8,3 & 7,5 & 15,2 & - & - & - & $\mathrm{Pm}_{4}$ & 7,1 & 3,9 & 9,3 & 6,7 & 4,1 & 9,3 \\
\hline $\mathbf{M}^{1}$ & 1,2 & 12,1 & 7,8 & - & - & - & $\mathbf{M}_{1}$ & 11,1 & 6,8 & 17,1 & 10,6 & 6,5 & $(16,1)$ \\
\hline $\mathbf{M}^{2}$ & 1,1 & 9,3 & 4,6 & - & - & - & $\mathrm{M}_{2}$ & 4,4 & 5,6 & 8 & - & - & - \\
\hline & & & & & & & $\mathbf{M}_{3}$ & - & - & - & - & & \\
\hline
\end{tabular}

TABLA 6

Medidas en milímetros de piezas dentales del zorro de Miraflores. 
un entierro primario ya que se conservaron huesos muy pequeños como falanges proximales, mediales y distales.

El zorro de Miraflores probablemente corresponde a una hembra, debido al tamaño y morfología de las inserciones musculares que se encuentran en los maxilares y las mandíbulas, de edad adulta (aprox. 7 años) (Tablas 6 y 7). Está muy claro su uso exclusivo como ofrenda, ya que solo se tomó en cuenta el cráneo y no presenta marcas antrópicas. El contexto donde fue depositado corresponde a un sitio periférico de filiación Tiwanaku, importante por su ubicación cerca del río Choqueyapu donde se explotaba oro. Por otra parte esta ofrenda de zorro estaba a pocos metros de otra que contenía la cabeza de un cóndor, animal que simboliza poder y conexión con el mundo de arriba (Mendoza, 2017).

\begin{tabular}{|c|c|c|c|c|}
\hline 11 & 12 & 14 & 19 & 20 \\
\hline 34,75 & 29,75 & 15,56 & $(15,80)$ & 13,35 \\
\hline
\end{tabular}

TABLA 7

Medidas en milímetros de la mandíbula izquierda del zorro de Miraflores.

Llama la atención que los tres individuos de zorro andino son de edad adulta mayor. Si existió una selección por edad, probablemente ésta tenga que ver con connotaciones simbólicas como la sabiduría y experiencia tal como sucede con la selección de autoridades en las Comunidades del Altiplano boliviano (Choque, 2004; Carrillo, 2015). Otra opción puede estar relacionada con la facilidad de caza de animales viejos por ser menos ágiles que los jóvenes.

\section{REPRESENTACIONES ICONOGRÁFICAS}

En época prehispánica, para el período Tiwanaku, son escasas las representaciones iconográficas de cánidos en comparación con las representaciones de félidos y camélidos, siendo más dificultosa, aún, la diferenciación entre perro y zorro andino. Mendoza (2004) planteó diferencias morfológicas para representaciones iconográficas naturalistas de perro y zorro, las cuales se resumen en la Tabla 8.

Las variables presentadas en la Tabla 8 nos muestran, primeramente, que las características morfológicas de ambas especies de cánidos responden a su condición de especie silvestre en el caso del zorro andino y doméstica en el caso del perro, por otra parte los artistas prehispánicos representaron al zorro andino con la posición anatómica, actitud y elementos iconográficos asociados para destacar un estatus y jerarquía diferente al perro. Esto contrastado con el análisis arqueofaunístico corrobora el manejo diferenciado reflejado en contextos ceremonial-doméstico en el período Formativo-Wancarani y ceremonial-ritual en el período Tiwanaku, en cambio el perro puede estar presente en diversos contextos en diferentes períodos y cumpliendo distintos roles (alimento, compañía y ofrenda, entre otros). Hasta ahora, en Bolivia, no se han reportado hallazgos de perros asociados a personajes humanos de élite (sacerdotes) y animales que simbolizan poder (cóndor) (Mendoza, 2004, 2013, 2014).

Los indicadores de un manejo ritual de la estatuilla de zorro de Akapana (Figura 4) se refieren principalmente al contexto que es la habitación de

\begin{tabular}{|c|c|c|c|c|c|c|c|c|}
\hline Cánido & $\begin{array}{c}\text { Cabeza } \\
\text { (posición) }\end{array}$ & Orejas & Hocico & Boca & Cuerpo & Cola & Actitud & $\begin{array}{c}\text { Posición del } \\
\text { individuo }\end{array}$ \\
\hline Zorro & $\begin{array}{l}\text { Recta o } \\
\text { elevada. }\end{array}$ & $\begin{array}{l}\text { Siempre } \\
\text { paradas }\end{array}$ & $\begin{array}{l}\text { Muy } \\
\text { pronunciado }\end{array}$ & Cerrada & $\begin{array}{l}\text { Simétrico, } \\
\text { semirobusto }\end{array}$ & $\begin{array}{l}\text { Siempre gruesa por } \\
\text { el abundante pelo, la } \\
\text { posición puede ser } \\
\text { horizontal o vertical, } \\
\text { nunca entre las piernas }\end{array}$ & $\begin{array}{l}\text { Imponente, } \\
\text { altiva, } \\
\text { segura }\end{array}$ & $\begin{array}{l}\text { Echado, } \\
\text { sentado. }\end{array}$ \\
\hline Perro & $\begin{array}{l}\text { Variada: } \\
\text { Recta, } \\
\text { hacia abajo, } \\
\text { elevada, } \\
\text { entre otras }\end{array}$ & $\begin{array}{l}\text { Pueden } \\
\text { ser } \\
\text { paradas, } \\
\text { dobladas, } \\
\text { caídas }\end{array}$ & $\begin{array}{l}\text { Puede ser } \\
\text { pronunciado, } \\
\text { medio o } \\
\text { corto. }\end{array}$ & $\begin{array}{l}\text { Generalmente } \\
\text { abierta, } \\
\text { algunas veces } \\
\text { mostrando } \\
\text { dientes } \\
\text { y/o lengua } \\
\text { (jadeando) }\end{array}$ & $\begin{array}{l}\text { Muy variado } \\
\text { dependiendo } \\
\text { de morfotipos: } \\
\text { simétrico, de } \\
\text { gran tamaño, } \\
\text { mediano, de } \\
\text { tamaño pequeño, } \\
\text { patas largas, } \\
\text { patas cortas, entre } \\
\text { otros. }\end{array}$ & $\begin{array}{l}\text { Variada: gruesa, } \\
\text { delgada, delgada al } \\
\text { inicio y se y se engrosa } \\
\text { llegando a la punta. } \\
\text { La posición puede ser } \\
\text { variada, horizontal, } \\
\text { vertical, enrollada, entre } \\
\text { las piernas, etc. }\end{array}$ & $\begin{array}{l}\text { Variada, } \\
\text { agresiva, } \\
\text { juguetona, } \\
\text { tranquila, } \\
\text { cansada, } \\
\text { entre otras }\end{array}$ & $\begin{array}{l}\text { Variada: } \\
\text { caminando, } \\
\text { echado, } \\
\text { parado. }\end{array}$ \\
\hline
\end{tabular}

TABLA 8

Diferencias morfológicas en representaciones iconográficas entre zorro andino y perro. 
la cima de la pirámide donde se ofrendó la estatuilla, la cual fue posterior al desmantelamiento de la estructura, por lo que pudo tener el propósito de rememorar la importancia de este tipo de espacio sagrado ocupado por un sacerdote tiwanakota (Mendoza, 2004). Por otra parte, la estatuilla presenta una especie de incisión leve que va de oreja a oreja como un collar que puede ser un elemento de sujeción indicando el intento de sometimiento o domesticación de este animal.

La tableta de rapé de Niño Korin (Figura 5), presenta elementos que indican estatus vinculado a un manejo ritual, como el bastón de mando con dos cabezas de cóndores opuestas a cada extremo, simbolizando poder y una relación con el mundo de arriba (López, 2007). De igual modo en las orejas del zorro se apoya una especie de corona segmentada la cual se extiende hasta el cuello, siendo este otro elemento que denota poder y estatus ${ }^{1}$ (Torres, 2004). En el cuerpo (lomo) se muestra una cabeza humana de perfil, la misma posee una especie de cabellera o sombrero que podría representar al personaje poderoso (López, 2007; Mendoza, 2017). Por debajo de las patas se encuentra una decoración geométrica escalonada que podría estar representando el Alajpacha (mundo de arriba) (Posnansky, 1945) o tal vez la pirámide de Akapana donde reside y lleva a cabo su actividad el sacerdote.

Las tabletas de rapé son parte de equipos psicotrópicos propiedad de shamanes que gozaron de cierto estatus y tratamiento especial dentro de la sociedad tiwanacota (Llagostera et al., 1988). Su iconografía puede representar el animal en el que se transforma el portador en un determinado ritual y en el proceso psicoactivo al utilizar los alucinógenos. Datos etnográficos indican que la mayoría de los shamanes después de haber consumido alucinógenos tornan a convertirse en animales (Furst, 1965,1967; Eliade, 1996).

\section{DATOS HISTÓRICOS}

Según Rostworowski (2009), citada en Pozzi-Escot et al. (2012), existía la representación

\footnotetext{
1 Según Wassen (1972) en el mismo sitio (Niño Korin) se encontró otro equipo psicotrópico proveniente de una tumba multifamiliar donde el hombre portaba en la cabeza una corona de oro, además de una bolsa con medicamentos y amuletos.
}

de una zorra muerta en la puerta del Santuario de Pachacamac, presencia corroborada con el dato del cronista Cieza de León que afirma que el templo de Pachacamac era importante porque solo se permitía el acceso a señores y sacerdotes que sacrificaban zorros como ofrendas.

Investigaciones etnohistóricas, etnográficas e iconográficas (arte rupestre), realizados por Huarita \& Taboada (2014), afirman que escritos coloniales de los siglos XVI y XVII identificaron el juego taptana ligado a un simbolismo religioso, donde en varios festejos se realizaba un baile ritual al zorro. Actualmente en muchas comunidades del altiplano boliviano se practica este juego siendo que el mismo está ligado a ritos agrícolas, donde el zorro (aullido) es el indicador del inicio de la fiesta de Guadalupe, del juego come taptana, la siembra y los cortejos, y con el nacimiento de las crías del zorro se cierra la primera fase de siembra, fiestas como la de San Andrés y los carnavales, entre otros acontecimientos. De esta manera se considera al zorro como un cánido sagrado, equilibrador de la vida social y agrícola (Huarita \& Taboada, 2014).

Crónicas del período Colonial tardío (Cobo, 1964; Garcilazo de la Vega, 1995) nos hablan del zorro como un animal ponzoñoso de mal agüero que da mala suerte si se cruza en el camino. Pero por otra parte, también es recurrente la relación que se le atribuye con la luna, el cielo (Itier, 1997) y también con animales como el cóndor (Callejas, 2015) y el oso (Choque \& Mamani, 1997) en mitos, cuentos y leyendas (Gemio, 1994).

\section{CONCLUSIONES}

Los restos arqueofaunísticos de tres individuos de zorro andino evidencian un manejo ritual debido al contexto doméstico-ceremonial (caso de Chuquiña) ya que se aprovechó, inicialmente, la piel del zorro, en cambio el caso del zorro de Miraflores indica un uso exclusivo como ofrenda. En el caso de Putuni, pudo ser utilizado como ajuar o acompañante de los individuos humanos enterrados.

El considerar solo el cráneo o solo las mandíbulas con el esqueleto postcraneal, indica una selección simbólica que puede responder a ciertas consideraciones ligadas a la importancia de las partes del cuerpo. Del mismo modo las tres representaciones iconográficas de zorros andinos, por su 
contexto, asociaciones y elementos iconográficos internos de cada una, evidencian que estas especies estuvieron ligadas a un personaje de alto estatus dentro del Estado Tiwanaku que pudo ser un sacerdote, de esta forma el manejo ritual se plasma en la representación de zorro que simboliza a este.

Los datos históricos confirman la sacralidad del zorro andino que va más allá de su uso como ofrenda, acompañante de los muertos, símbolo de estatus y nexo con el mundo de arriba, el zorro es un importante indicador agrícola, demarcador de tiempos rituales que van acompañados de juegos y fiestas importantes.

Esta concepción se fue transformando en época colonial, considerando al zorro como un animal de mal agüero, depredador del ganado introducido por los europeos. Es así que podemos afirmar que el zorro andino fue en un principio adorado y después rechazado.

\section{AGRADECIMIENTOS}

A la comunidad de Chuquiña, al Museo Regional de Tiwanaku, al Museo Nacional de Arqueología por el préstamo de piezas, a los arqueólogos Rubén Mamani, Karen Mamani, Ruden Plaza, Luis Viviani, Carlos Lemuz, al antropólogo Genaro Huarita, a la Conservadora Irene Delaveris por su gran colaboración y a los organizadores de la III Reunión Académica del Grupo de Zooarqueología Neotropical que hicieron las correspondientes gestiones para la publicación de las ponencias en esta prestigiosa revista. Este artículo está dedicado a mi querido amigo Christopher Götz, †.

\section{REFERENCIAS}

Albarracín-Jordán, J.; Capriles, J. \& Miller, M. 2014: Transformations in ritual practice and social interaction and the Tiwanaku periphery. Antiquity Publication Ltd. 88: 851-862.

Blanco, A.; Rodríguez, B. \& Valadez, R. 2009: Estudio de los cánidos arqueológicos del México prehispánico, Textos básicos y manuales, Instituto Nacional de Antropología e Historia, Universidad Nacional Autónoma de México, Instituto de Investigaciones Antropológicas, México.
Callejas, N. 2015: El Zorro y el Cóndor [You Tube. MKV]. Recuperado de: www.google.com/url?sa=t\&source $=$ web $\&$ rct $=\mathrm{j} \& u r l$.

CAPriles, J. 2017: Arqueología del pastoralismo temprano de camélidos en el Altiplano central de Bolivia. Plural, IFEA, La Paz.

CARrillo, J. 2015: Estructuras y Jerarquías en las Comunidades de Cabildo de Calamarca. Temas Sociales 36: 57-85.

Choque, E. 2004: Las prácticas de poder y liderazgo de los Jilaqatas y Mama t'allas en Huachacalla Marka. Tesis para optar al título de Magister en Educación Intercultural Bilingüe con la mención de Gestión en Planificación. Facultad de Humanidades y Ciencias de la Educación. Universidad Mayor de San Simón. Cochabamba. Ms.

Choque, M. \& Mamani, C. 1997: El pícaro zorro y el oso. THOA Aruwiyiri, La Paz.

Coвo, B. 1964: Obras del Padre Bernabé Cobo. Historia del Nuevo Mundo. Biblioteca de Autores Españoles, Madrid.

Cordero, G. 1967: Valioso testimonio arqueológico en Niño Korin-Charasani. Khana Arte y Letras Revista Municipal 38: 139-144.

De Mello, B. \& Zuercher, G. 2005: Speothos venaticus. Mammalian Species 786: 1-6.

Deustua, I. 2008: Descripción de los conflictos, percepciones, actitudes y usos tradicionales que poseen los pobladores rurales del Departamento de Ayacucho, Provincia de La Mar, Distrito de Anco sobre los mamíferos altoandinos (Orden Carnívora) durante el año 2006. Tesis de Grado Biólogo. Facultad de Ciencias, Departamento de Biología, Universidad Nacional Agraria La Molina. Ms.

DrIesch, A. von den 1976: A guide to measurement of animal bones from archaeological sites. Peabody $\mathrm{Mu}-$ seum Bulletin 1. Peabody Museum of Archaeology and Ethnology, Harvard University.

Eliade, M. 1996: El chamanismo y las técnicas arcaicas del éxtasis. Fondo de Cultura Económica, Madrid.

Furst, P. 1965: West Mexican Tomb Sculpture as Evidence for Shamanism in Prehispanic Mesoamerica. Antropológica 15: 29-80.

- 1967: The Olmec Were-Jaguar Motif in the Light of Ethnographic Reality. In: Benson, E. (ed.): Conference on the Olmec: 143-178. Dumbarton Oaks Research Library and Collections, Trustees for Harvard University, Washington D.C.

Garcilazo De La Vega, I. 1995: Comentarios Reales de los Incas. Tomos I-II. Edición, índice analítico y glosario de Carlos Aranibar. FCE. México. 
Gemio, L. 1994: Tiwulax kunans atipkaspapi (el zorro no puede vencer nunca) una lectura de los cuentos del zorro. Educación Bilingüe e Intercultural, Tomo I. RAE, La Paz.

GOEPFERT, N. 2004: Ofrendas y Sacrificios animales en la cultura Mochica: El ejemplo de la Plataforma Uhle, complejo arqueológico Huacas del Sol y de la Luna. En: Castillo, L.; Bernier, H.; Lokcard, G. \& Rucabado, J. (eds.): Arqueología Mochica Nuevos Enfoques: 231-244. Instituto Francés de Estudios Andinos. Universidad Católica del Perú, Lima.

Guzmán, J., D’Elía J. \& Ortiz, J. 2009: Variación geográfica del zorro Lycalopex culpaeus (Mammalia, Canidae) en Chile: implicaciones taxonómicas. Revista de Biología Tropical.57 (1-2): 421-432.

Hocquenghem, A. 1987: Iconografía Mochica. Universidad Católica del Perú, Lima.

Huarita, G. \& Taboada, F. (2014): Come taptana: Persistencia histórica de un juego de tablero indígena que presagia el ciclo agrícola en el altiplano boliviano. En: Málaga, A.; Díaz, A. \& Rosas, C. (eds.): IX Congreso Internacional de Etnohistoria. Universidad de Tarapacá, Chile.

ITIER, C. 1997: El zorro del cielo: Un mito sobre el origen de las plantas cultivadas y los intercambios con el mundo sobrenatural. Boletín Francés de Estudios andinos 26(3): 307-346.

LemuZ, C. 2016: Informe de excavaciones en Torre 24, Avenida Busch, casi Plaza Busch Proyecto de Mitigación de impactos arqueológicos en la Avenida Busch y las Plazas Busch y San Martin-Triangular, en el Marco de la Construcción de la Línea Blanca del Teleférico de la ciudad de La Paz. CPM Investigación \& Desarrollo. Empresa contratante: Doppelmayr Bolivia S.A. Ms.

Llagostera, A., Torres, M. \& Costa, M. 1988: El complejo psicotrópico en Solcor-3 (San Pedro de Atacama). Estudios Atacameños 9: 61-80.

LóPEZ, M. 2007: Interpretación simbólica de la iconografía del sacrificador y el señor de los cetros: una visión desde los mitos. Memoria para optar al título de arqueóloga, Departamento de Antropología Arqueología, Facultad de Ciencias Sociales, Universidad de Chile. Ms.

Lucherini, M. \& Luengos, E. 2008: Lycalopex gymnocercus (Carnívora: Canidae). Mammalian Species 820: $1-9$.

ManZanilla, L. 1992: Akapana. Una pirámide en el centro del mundo. UNAM. IIAA. México D.F.

Meadow, R. 2000: The contributions of Barbara Lawrence to the study of dogs, with a comprehensive listing of her measurement definitions». In: Proceedings of the $1^{\text {st }}$ ICAZ Symposium on the History of the Domestic Dog: 35-50. Victoria, B.C.

MendozA, V. 2004: El perro en las sociedades andinas del pasado: Un aporte arqueozoológico. Tesis de Licenciatura en Arqueología. Facultad de Ciencias Sociales, Universidad Mayor de San Andrés. La Paz. Ms.

- 2013: El Perro Prehispánico Boliviano. Su historia a través de la Arqueología. 3V Gráficos, La Paz.

- 2014: Evidencia de uso del perro (Canis lupus familiaris) en un contexto ritual (Tiwanaku, Bolivia). Revista Chilena de Antropología 30: 104-108.

- 2017: Informe Preliminar del Análisis Arqueozoológico del Proyecto Arqueológico Miraflores (Construcción de la Línea Blanca de mi Teleférico). Presentado al Proyecto Arqueológico Miraflores PAM. Ms.

Mercado, I. \& Millares, J. 1999: Mamíferos. En: Forno, E. \& Baudoin, M. (eds.): Historia Natural de un Valle en los Andes: 293-344. Instituto de Ecología, Facultad de Ciencias Puras y Naturales, UMSA, La Paz.

Oblitas, E. 1978: Cultura Callawaya. Ed. Populares Camarlinghi, La Paz.

PaZ, J.L.; García, R.; Jiménez, N.; VARgas, J.; CÁCERES, O.; Fontenla, R.; Quispe, O.; SeJas, C.; Ticona, M.; UlloA, D. \& Villegas, V. 2008: La presencia Tiwanaku en el sitio de Ach-10 (Valle de Achocalla, Bolivia). En: Rivera, C. (ed.): Arqueología de las tierras altas, valles interandinos y tierras bajas de Bolivia: 169-186. Memorias del I Congreso de Arqueología de Bolivia. IIAA, PIEB, ASDI-SAREC, La Paz.

PlazA, V. 1998: Informe de excavaciones en la Comunidad de Qiwaya, Proyecto «Chullpa Pacha 98», noviembre. La Paz. Ms.

Pérez Ripoll, M. \& Morales Pérez, J. 2008: Estudio tafonómico de un estudio actual de huesos de Vulpes vulpes y su aplicación en la Zooarqueología. En: Diez, J. (ed.): Zooarqueología hoy, Encuentros Hispano-Argentinos: 179-189. Universidad de Burgos.

Ponsnansky, A. 1945: Tiahuanacu, la cuna del hombre americano. Vols. I y II. J.J. Augustin Publisher, New York.

Pozzi-Escot, D.; Cornejo, I.; Angulo, E. \& Bernuy, K. 2012: Estudio preliminar de los hallazgos de Canis familiaris en la Pirámide con Rampa No7, Santuario de Pachacamac, Perú. Revista del Museo de Antropología 5:171-184.

Torres, C. 2004: Imágenes legibles: la iconografía Tiwanaku como significante. Boletín del Museo Chileno de Arte Precolombino 9: 57-74.

VALADEZ, R. 1996a: Estudio del perro mesoamericano. AMMVEPE 3:11-17. 
- 1996b: Anatomía dental del perro pelón mexicano. Estudio odontológico comparativo del Xoloitzcuintle. AMMVEPE 5: 179-183.

Viviani, L. \& Callisaya, L. 2009: Aproximaciones a la dinámica social y espacial en el templo de Putuni, Tiwanaku. Informe Técnico Proyecto Arqueológico Putuni. Ms.

Wallace, R.; Alfaro, F.; Sainz, L.; Ríos-Uzeda, B. \& Noss, A. 2010: Canidae. En: Wallace, R.; Gómez, H.; Porcel, Z. \& Rumiz, D. (eds.): Distribución, ecología y conservación de los mamíferos medianos y grandes de Bolivia: 367-400. Centro de Ecología Difusión Simón I. Patiño. Santa Cruz de la Sierra. Bolivia.

WASSÉn, S. 1972: A medicine-man's implements and plants a Tihuanacoid Tmb in Highlands Bolivia. Göteborgs Etnografiska Museum. Göteborg.

WING, E. 1989: Human use of canids in the central Andes. In: Eisenberg, J. \& Redford, K. (eds.): Advances in neotropical mammalogy: 256-278. Sandhill Crane Press, Gainesville. 